\title{
A Demonstration of Adaptive Spectrum Sensing and Interference Suppressed Opportunistic Secondary Transmission
}

\author{
Samson Sequeira*, Srinivas Pinagapany*, Yasunori Futatsugi ${ }^{\dagger}$, Masayuki Ariyoshi ${ }^{\dagger}$, Predrag Spasojevic* \\ *WINLAB, ECE Department, Rutgers University \\ ${ }^{\dagger}$ System Platforms Research Laboratories, NEC Corporation
}

\begin{abstract}
We consider spectrum sensing and Non-Contiguous OFDM (NC-OFDM) with interference avoidance to facilitate opportunistic secondary transmission. In this application protecting an incumbent primary user of the spectrum from secondary emissions is a key design requirement. We present an adaptive sense-and-carefully-transmit system which integrates a spectrum sensing module with an NC-OFDM transmitter having a powerful interference avoidance capability. The spectrum sensing module uses a rank-order filtering (ROF) algorithm to automatically set the detection threshold. The interference suppression capability of the NC-OFDM transmitter is enhanced using a technique called Interference Avoidance using Partitioned Frequency and Time domain processing (IA-PFT). We have implemented our Dynamic Spectrum Access (DSA) protocol using the GNU RadioUSRP2 platform. We demonstrate the DSA system performance by experiments performed in the ORBIT Testbed, WINLAB, Rutgers University.
\end{abstract}

\section{INTRODUCTION}

Opportunistic spectrum access of the licensed band by unlicensed devices is being considered as a potential solution to the spectrum scarcity crisis. This approach requires that the incumbent primary users be protected from detrimental interference from the opportunistic secondary users. A parallel problem in this scenario is that the performance of secondary users must also be optimized, since they do not have a dedicated bandwidth and have to overcome the interference from the primary users. This is highlighted in Figure 1. Once these initial technical challenges are resolved, a wide variety of applications can be realized using DSA such as providing augmented coverage in the existing cellular network, rural broadband access, ad-hoc mesh networks for military, medical and emergency applications, internet backhaul traffic etc.

Spectrum sensing to enable secondary spectrum access is an extensively researched topic. Various sensing techniques such as energy detection, matched filter detection and cyclostationary feature detection have been considered in this setting [1]. Conventional energy detection approaches rely on prior knowledge of noise power in the bandwidth of interest. Measuring the noise power requires taking the system offline for calibration purposes [2]. In addition to this being infeasible in realistic scenarios, calibrating a system this way is an extensive process. The thermal variations and aging of components cause the noise floor of the receiver to drift significantly.

Several implementations of Dynamic Spectrum Access have employed NC-OFDM to facilitate reconfigurable secondary spectrum allocation [3], [4]. The NC-OFDM system is a modified OFDM transceiver with non-contiguous occupied frequencies. This enables the creation of spectral notches in the

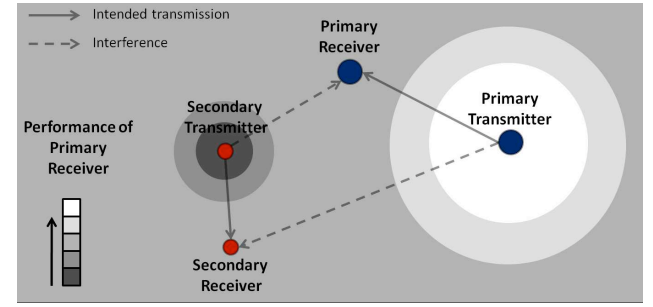

Fig. 1. Typical Primary-Secondary Co-existence Scenario

transmitted spectrum to avoid interference to the primary user. However, the occupied frequencies in a NC-OFDM transmitter will cause spectral leakage into the notch which adversely affects the primary users.

We present an adaptive sense-and-carefully-transmit system which integrates a spectrum sensing module with an NCOFDM transmitter having a powerful interference avoidance capability. The spectrum sensing module uses a rank-order filtering (ROF) algorithm to automatically set the detection threshold. The interference suppression capability of the NCOFDM transmitter is enhanced using a technique called Interference Avoidance using Partitioned Frequency and Time domain processing (IA-PFT) [5]. We have implemented our Dynamic Spectrum Access (DSA) protocol using the GNU Radio-USRP2 platform. We demonstrate the DSA system performance by experiments performed in the ORBIT Testbed, WINLAB, Rutgers University.

Section II discusses the top level system setup. Section III describes the ROF based sensing and IA-PFT based secondary transmitter. Section IV describes the demonstration setup and the GNU Radio-USRP2 implementation. Section V illustrates the results of the DSA experiment in the ORBIT testbed. Section VI concludes.

\section{SySTEM MODEL}

\section{A. Top-level System Setup}

The top level system model is represented by Figure 2, which depicts a single primary transmitter along with a secondary transmitter, secondary receiver and the spectrum sensing module.

The system has the following three main functional units:

1) Primary transmitter: The primary transmitter is a Wireless Microphone signal which has been emulated using a Vector Signal Generator (VSG).

2) Secondary Transmitter: The secondary transmitter is the crux of our system and consists of the spectrum 


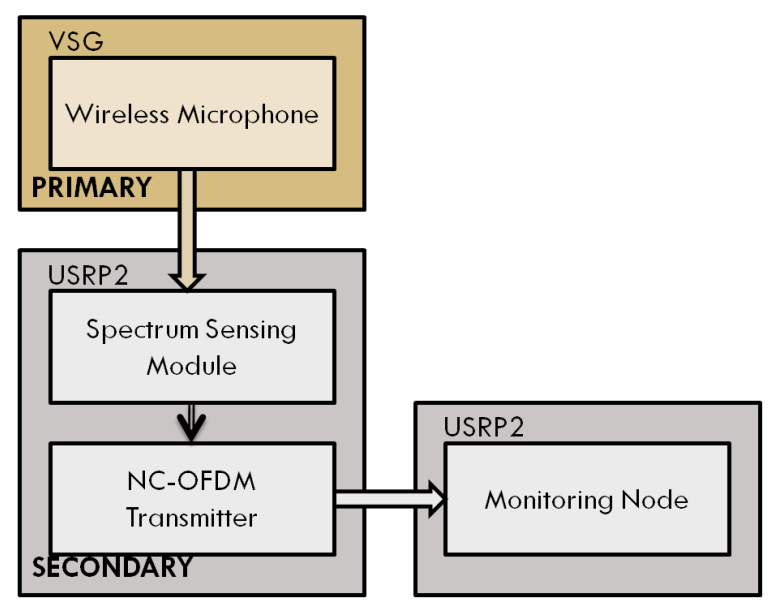

Fig. 2. System Setup

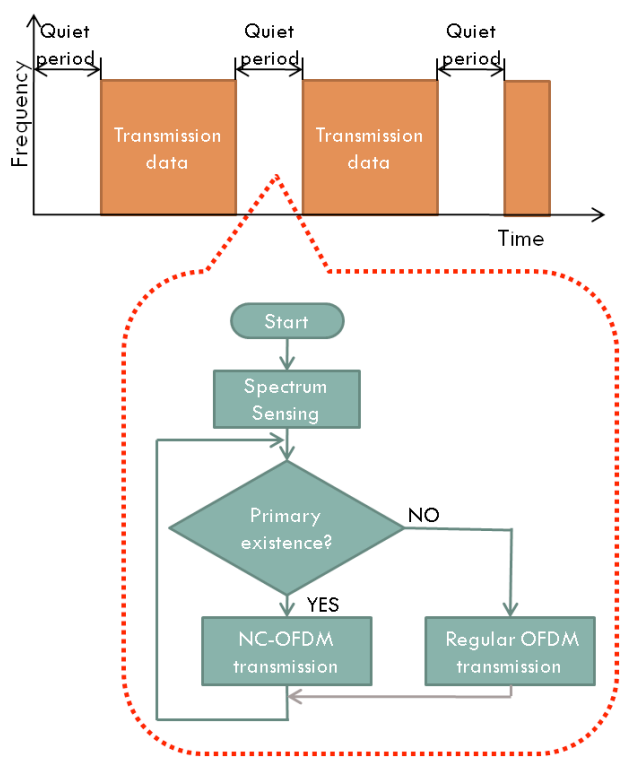

(a)

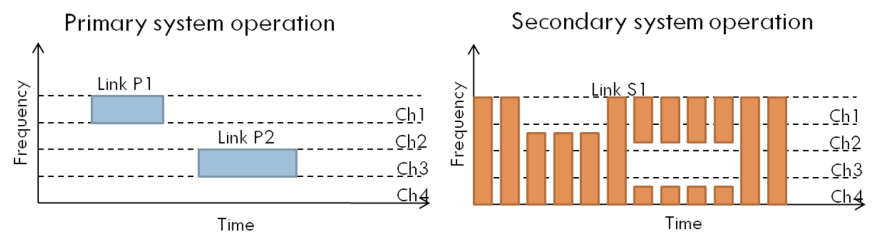

(b)

Fig. 3. DSA protocol timeline

sensing module and a NC-OFDM based transmitter with Interference Avoidance capability.

3) Monitoring Node: This USRP2 node is used to record the RF transmissions in addition to a spectrum analyzer.

\section{B. DSA Protocol}

Figure 3(a) illustrates the timeline of our DSA protocol. The process begins with sensing the desired spectrum to determine if the primary transmitter is $\mathrm{ON}$ and its spectrum information i.e. carrier frequency and bandwidth. Spectrum

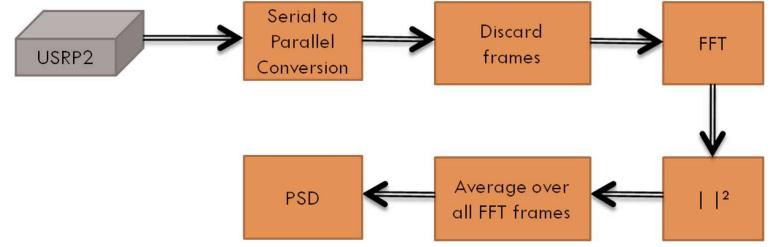

Fig. 4. Spectrum Sensing Implementation

sensing uses an adaptive threshold is calculated based on the automatic estimation of the instantaneous noise floor achieved by means of ROF algorithm. During this period of sensing the secondary does not employ any transmissions since it is in reception mode and hence it is referred to as the quiet period. If the primary transmitter is not detected, we proceed with OFDM transmission which occupies the entire bandwidth of interest. If a primary transmitter is detected we employ NCOFDM transmission which has subcarriers deactivated at those frequencies occupied by the primary transmitter as illustrated in Figure 3(b).

Choosing the duration of the secondary transmission is a key design issue. It should depend on the type of primary transmission. The primary transmission could be continuous or intermittent depending on the type of application. If the secondary transmission duration chosen is too long then there are chances that the primary transmitter might come $\mathrm{ON}$ during this period. On the other hand if this duration is too short the secondary system would spend a larger fraction of time in the sensing operation thereby not achieving maximum efficiency possible.

\section{Spectrum Sensing AND Interference AVOIDANCE ALGORITHMS}

\section{A. ROF Based Spectrum Sensing}

The system level implementation of the algorithm is shown in Figure 4. The noise floor changes continuously with frequency, and hence, the noise floor needs to be computed onthe-fly. We consider the technique of automatically estimating the noise floor as presented in [6]. The authors have applied binary morphological processing and have suggested ROF approach as an alternative. Figure 5 shows a brief overview of the algorithm used for estimating the noise floor. This algorithm is called the ROF algorithm. It involves operating on the PSD vector with consecutive iterative steps called erode(opening) and dilate(closing). These steps are repeated until the change in the noise floor is small compared to that in the previous iteration. The spectrum sensing module observes the RF spectrum, calculates the PSD and provides a SensorMask to the transmitter, indicating which frequencies are available.

\section{B. Interference Avoidance Secondary Transmission}

We implemented the following two types of secondary transmitters:

1) Regular NC-OFDM transmitter

2) IA-PFT enabled NC-OFDM transmitter

The secondary transmitters construct an appropriate CarrierMask to be used for transmission. The sub-carrier mapper block activates and deactivates respective tones in 


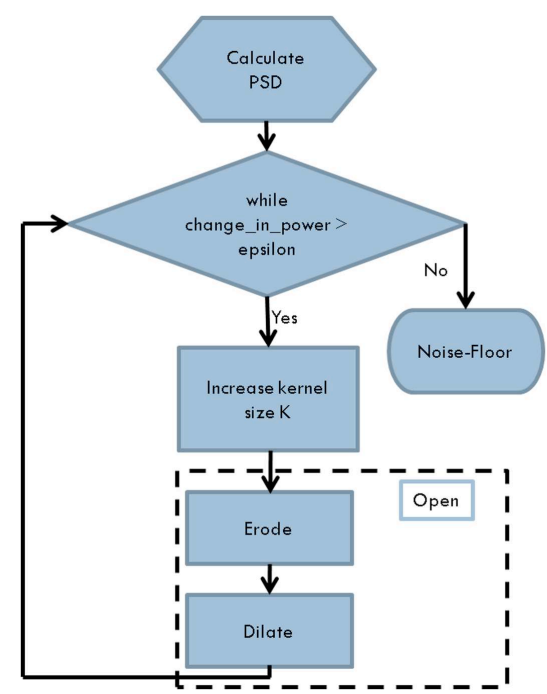

Fig. 5. Noise Floor Estimation Algorithm/ROF Algorithm

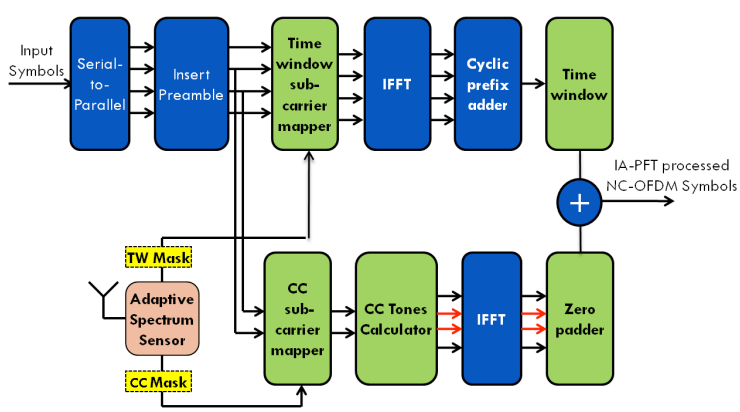

Fig. 6. IA-PFT enabled NC-OFDM secondary transmitter

NC-OFDM based on the carrier-mask. During a regular NCOFDM transmission, energy is leaked into the notches. This energy is above the average noise floor in the band and can hamper the operation of the wireless microphone. Hence to suppress this leakage we employ the IA-PFT technique [5] which achieves enhanced interference suppression.

In IA-PFT we process the information symbols in two streams, namely Time Windowing (TW) and Cancellation Carriers (CC), as illustrated in the block diagram of IA-PFT enabled NC-OFDM transmitter in Figure 6. These two streams are generated by TW mapper and CC mapper blocks which use the carrier-mask to determine the position of the notch. Once the notch is determined, the TW mapper deactivates $Q$ subcarriers on either side of the notch. The CC mapper generates a symbol stream with complementary sub-carrier map having only the $Q$ sub-carriers on the outer edges of the notch. The streams are then processed independently by the TW and the CC blocks. Time windowing involves pulse shaping the NCOFDM symbols with a raised cosine filter and the tail from the current NC-OFDM symbol is added to cyclic prefix of the next NC-OFDM symbol. This operation is performed on the upper symbol stream shown in Figure 6. Cancellation Carrier insertion involves adding two CC tones to the extreme ends of the notch to cancel the leakage due to the $Q$ active sub-carriers next to the edges of the notch. IFFT is performed over the CC symbols to generate the lower NC-OFDM symbol stream in Figure 6. In this stream, only the $\mathrm{CC}$ tones and the sub-carriers

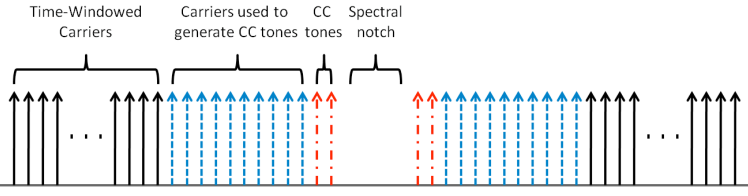

Fig. 7. IA-PFT processed NC-OFDM symbols at the output of the adder

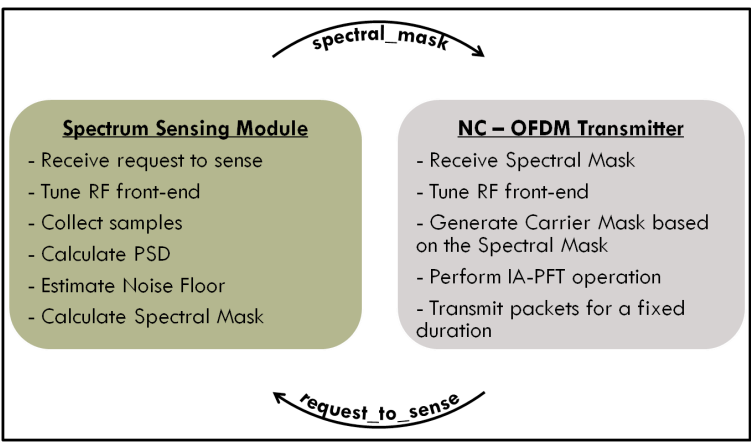

Fig. 8. Handshaking between the sensing and transmitting modules

needed to calculate $\mathrm{CC}$ are active. As a result, the TW and CC streams are complementary in nature. The CC processed NCOFDM symbols are zero padded to match the length of the TW processed symbols. The symbols from CC stream and the TW stream are combined by the adder to result into symbols illustrated in Figure 7. The output of the adder is the IAPFT processed NC-OFDM symbols that provides additional interference suppression to the regular NC-OFDM transmitter.

\section{A GNU RADIO - USRP2 ImPLEMENTATION}

The spectrum sensing module and secondary transmitter applications are implemented in a single physical unit which comprises of a Linux based host computer and a commercially available hardware unit called the Universal Software Radio Peripheral ver. 2 (USRP2) [7]. USRP2 uses the XCVR2450 $\mathrm{RF}$ daughter board which is a $2.4-2.5 \mathrm{GHz}$ and 4.9 to 5.85 $\mathrm{GHz}$ dual-band transceiver with $20 \mathrm{dBm}$ max transmit power in the $2.4 \mathrm{GHz}$ band and $17 \mathrm{dBm}$ in the $5 \mathrm{GHz}$ band. The GNU Radio software development toolkit, an open source software with source code written in Python and $\mathrm{C}++$ [8], provides the necessary signal processing modules to implement the software radio. The handshaking between the sensing and secondary transmitter modules is described in Figure 8.

We have implemented our system referring the LTE specifications [9],[10]. The total bandwidth scanned by the spectrum sensor is $3.84 \mathrm{MHz}$ using an FFT size of 256 resulting in a bin resolution of $15 \mathrm{kHz}$. The secondary bandwidth is 2.16 $\mathrm{MHz}$ which is divided into 12 resource blocks (RB) with 12 frequency bins per RB. This is illustrated in Figure 9. The primary signal occupies RB 3 in this example setup. In addition RB3, RB2 and RB4 are also notched by the secondary to ensure the interference suppression. The number of deactivated sub-carriers for IA-PFT is set as $Q=10$ in our setup.

Our demonstration setup is shown in Figure 10 where the primary transmitter, secondary transmitter and the monitor are placed at different locations in the ORBIT grid.

A vector signal generator (VSG) is used to transmit the wireless microphone signal. The wireless microphone signal is a frequency modulated signal whose tone frequency and 


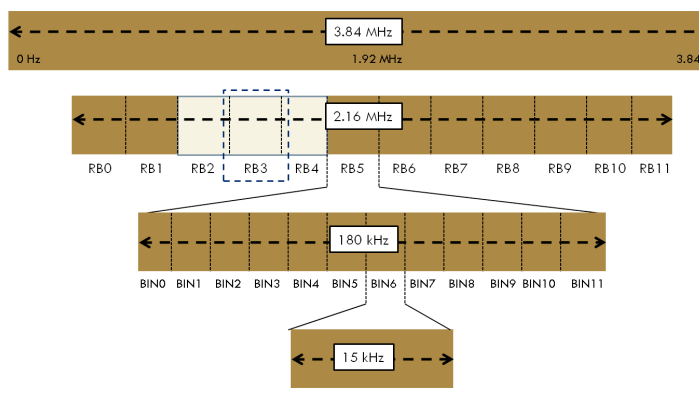

Fig. 9. Spectrum Diagram

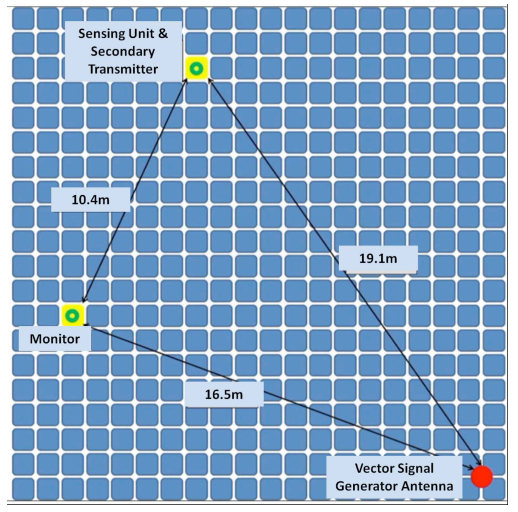

Fig. 10. Experimental Setup

frequency deviation depend on the mode of operation. The four modes of operation are [11]:

1) Off

2) Silent

3) Soft speaker

4) Loud speaker

The generated signal is shown in Figure 11. The bandwidth of the signal is $200 \mathrm{kHz}$ and the transmit power is $-30 \mathrm{dBm}$ and hence it occupies a little bit more than one RB.

The noise floor is estimated over the scanned bandwidth using the ROF algorithm. The threshold for each RB $i$ is calculated as follows:

threshold $_{i}=$ noise_floor $_{i}+x$

where $x$ is a small increment that determines the probability of false alarm and missed detection. Using the thresholds, a 12 bit vector is generated corresponding to the $12 \mathrm{RBs}$. A resource block is considered to be occupied if the power in it exceeds its threshold . $_{\text {. }}$

NC-OFDM transmitter allows the following parameters to be changed by the user:

- Center frequency $f_{c}$ from $(2.4-2.5 \mathrm{GHz}$ and $4.9-5.85$ $\mathrm{GHz})$

- FFT size $N_{F F T}$ from (4 - 4096)

- Carrier mask string

- Interpolation/Decimation (4 - 512)

- Transmit amplitude $(100+\mathrm{mW}$ output on $2.4 \mathrm{GHz}$, $50+\mathrm{mW}$ output on $5 \mathrm{GHz}$ maximum and scaled between 0 and 1)

- Modulation (BPSK, QPSK, 16QAM)

- Samples-per-symbol (2 - 7)

- CP length

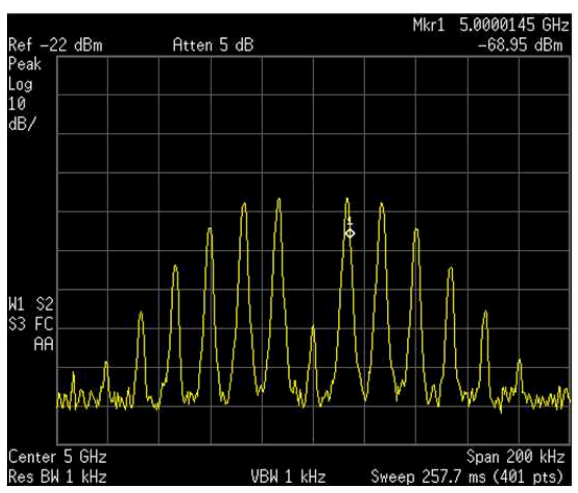

Fig. 11. Wireless Microphone Signal on a spectrum analyser

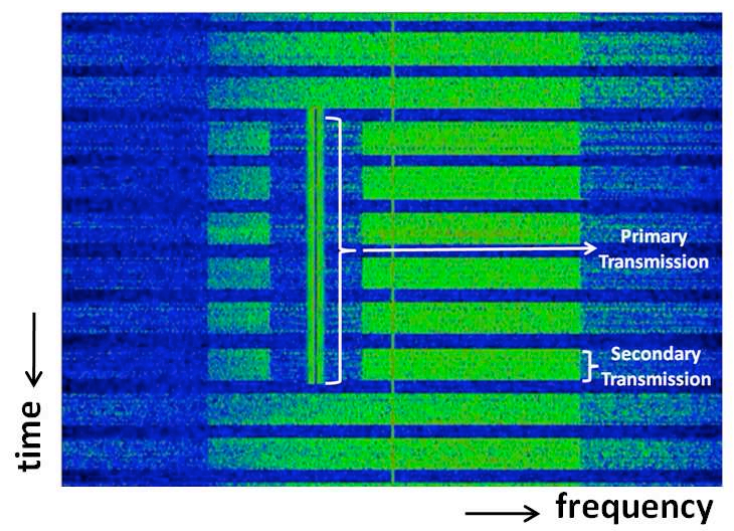

Fig. 12. DSA operation observed on the spectrum analyzer

\section{DSA DEMONSTRATION}

Figure 12 shows the spectrogram of the DSA operation. The wireless microphone signal in this case is $\mathrm{ON}$ for 5 secs and OFF for 5 secs. Secondary transmitter sends out packets for a fixed duration of time between the sensing durations which is a design parameter. We verified experimentally that the ROF based sensing technique correctly estimates the noise floor and fixes a detection threshold automatically. The simulation results demonstrating its performance is illustrated in Figure 13.

We also collect samples from over-the-air transmission of IA-PFT enabled NC-OFDM secondary system at the monitoring node and plot its power spectral density. It is evident from the plot in Figure 14 that there is about $10 \mathrm{~dB}$ of additional suppression as compared to regular NC-OFDM.

A video of the demonstration can be found at [12].

\section{CONCLUding REMARKS}

We present a GNU Radio implementation of an adaptive sense-and-carefully-transmit system which integrates an ROF based spectrum sensing module with an IA-PFT enabled NCOFDM transmitter having a powerful interference avoidance capability. We verified our DSA protocol experimentally in the ORBIT testbed. We found that the ROF based sensing technique correctly estimates the noise floor and automatically fixes a detection threshold. The NC-OFDM secondary transmitter uses only some of its sub-carriers when the wireless microphone is ON thereby avoiding interference to the wireless 


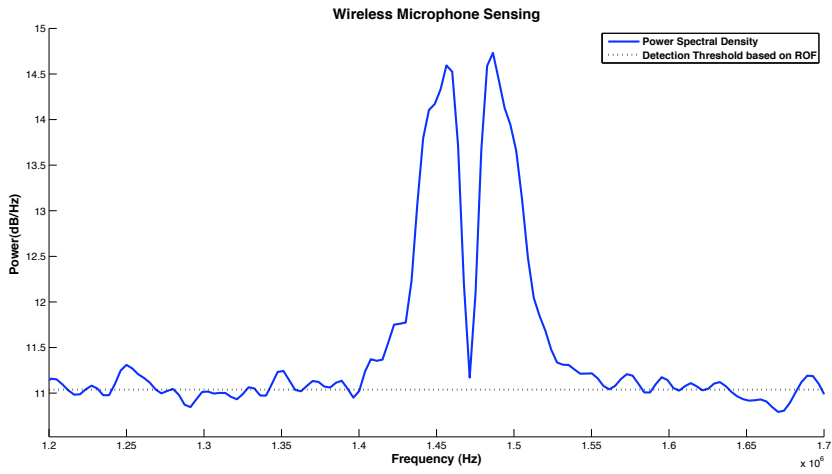

[8] GNU Radio http://www.gnuradio.org

9] Dr. Wes McCoy, Overview of the 3GPP Long Term Evolution Physical Layer

[10] 3GPP, TS 36.211 (V9.1.0), Physical channels and modulation March 2010

[11] C. Clanton, M. Kenkel, and Y. Tang, Wireless Microphone Signal Simulation Method IEEE 802.22-07/0124r0, March 2007

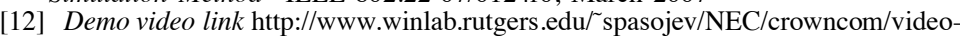
demo

Fig. 13. ROF based sensing for a wireless microphone signal

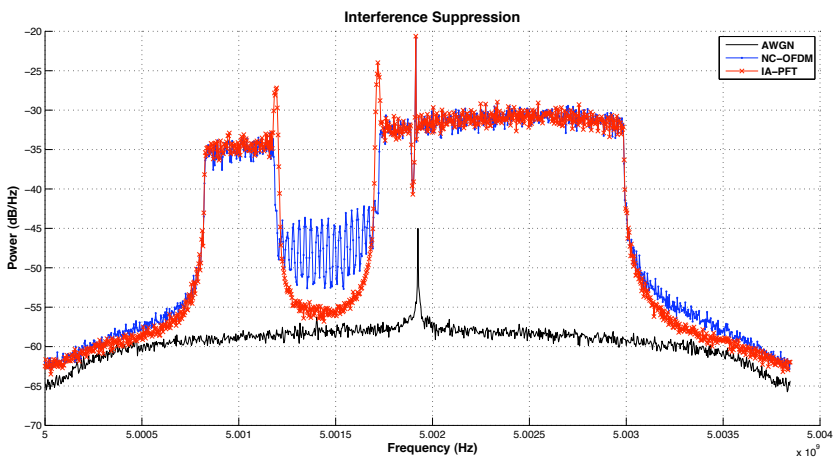

Fig. 14. Power Spectral Density of the IA-PFT enabled NC-OFDM transmission

microphone. There is about $10 \mathrm{~dB}$ of additional suppression when IA-PFT is employed as compared to the regular NCOFDM in the GNU Radio implementation.

\section{ACKNOWLEDGMENT}

We would like to thank Ivan Seskar for helping us throughout the project with the experimental setup and the demo. We also thank Abhishek Bindiganavile for helping us organize the figures.

\section{REFERENCES}

[1] S. J. Shellhammer, Spectrum Sensing in IEEE 802.22 CIP2008, Santorini, Greece, 9-10 June 2008

[2] D. Cabric, A. Tkachenko, and R. W. Brodersen, Experimental study of spectrum sensing based on energy detection and network cooperation In Proceedings of the first international workshop on Technology and policy for accessing spectrum (TAPAS 2006), ACM, New York, USA

[3] J. D. Guffey, A. M. Wyglinski, and G. J. Minden, Agile Radio Implementation of OFDM Physical Layer for Dynamic Spectrum Access Research IEEE Global Telecommunications Conference, GLOBECOM '07.

[4] Z. Yan, Z. Ma, H. Cao, G. Li, W. Wang, Spectrum Sensing, Access and Coexistence Testbed for Cognitive Radio using USRP 4th IEEE International Conference on Circuits and Systems for Communications, ICCSC 2008.

[5] Y. Futatsugi and M. Ariyoshi, A Study on Interference Suppression Technique by Partitioned Frequency- and Time-domain Processing for Dynamic Spectrum Access IEICE Tech. Rep., vol. 110, no. 41, SR20109, pp. 49-55, May 2010.

[6] Ready, M.J., Downey, M.L., and Corbalis, L.J, Automatic noise floor spectrum estimation in the presence of signals Proceedings of ThirtyFirst Asilomar Conference on Signals, Systems \& Computers, vol.1, no., pp. 877-881 vol.1, Nov 1997.

[7] Universal Software Radio Peripheral http://www.ettus.com 